Conclusions: Rituximab is a safe and effective alternative to cyclophosphamide in the primary therapy of skin and lung manifestations of scleroderma.

Disclosure of Interest: None declared

DOI: 10.1136/annrheumdis-2018-eular.3464

\section{THU0407 EVALUATION OF SOLUBLE AND MEMBRANE HLA-G IN PATIENTS WITH SYSTEMIC SCLEROSIS AND ROLE OF THESE MOLECULES IN THE PATHOGENESIS OF THE DISEASE}

G. Murdaca, P. contini, S. Negrini, M. Borro, F. Puppo. Internal Medicine, University of Genova, Genova, Italy

Background: Systemic sclerosis (SSc) is a complex disease characterised by immune abnormalities, vascular damage and fibrosis. Human leukocyte antigen$\mathrm{G}(\mathrm{HLA}-\mathrm{G})$ is a non-classic class I major histocompatibility complex (MHC) molecule expressed on different cell lineages in both physiological and pathological conditions and detectable in soluble forms (sHLA-G1 and HLA-G5 shed and secreted isoform, respectively). Several immunomodulatory functions have been attributed to both membrane-bound and soluble HLA-G molecules. HLA-G is expressed on extravillous cytotrofoblast, in placenta but also in a few normal tissues, solid tumours, transplanted organs and virally infected cells. Soluble form (sHLA-G) derives from shedding of cleaved surface isoforms (sHLA-G1) or secretion of soluble isoforms (HLA-G5). Immunosuppressive functions have been attributed to both membrane HLA-G (mHLA-G) and sHLA-G.

Objectives: The aims of the present study were: 1) to determine the serum levels of sHLA-G molecules in a cohort of SSc patients with the limited or diffuse form of the disease; 2) to correlate sHLA-G levels with TGF- $\beta$; 3) to evaluate the expression of HLA-G in peripheral blood mononuclear cells (PBMC).

Methods: Thirtyfive patients (28 females/7 males, age 40-89 years) with diffuse SSc (dSSc, n. 12) or limited SSc (ISSc, n. 23) and 40 healthy sex and age matched controls were enrolled. Plasma sHLA-G, sHLA-G1 and HLA-G5 levels were determined by immunoenzymatic assays. $\mathrm{mHLA}-\mathrm{G}$ expression in peripheral blood mononuclear cells (PBMC) was evaluated by flow cytometry.

Results: The plasma levels of sHLA-G were higher in SSc patients (444.27 $\pm 304.84 \mathrm{U} / \mathrm{ml}$ ) compared to controls $(16.74 \pm 20.58 \mathrm{U} / \mathrm{ml})(p<0.0001)$. The plasma levels of TGF- $\beta$ were higher in SSc patients $(18937 \pm 15217 \mathrm{pg} / \mathrm{ml})$ compared to controls $(11099 \pm 6081 \mathrm{pg} / \mathrm{ml} ; \mathrm{p}=0.003)$ and a significant correlation was found between TGF $\beta$ and the plasma levels of total sHLA-G ( $r: 0.65 ; p<0.01)$, sHLA-G1 ( $r: 0.60 ; p=0.003)$ and HLA-G5 ( $r: 0.47 ; p=0.02)$. The percentage of HLA-G-positive monocytes $(0.98 \pm 1.72), \mathrm{CD} 4+(0.37 \pm 0.68), \mathrm{CD} 8+(2.05 \pm 3.74)$ and CD4 +CD8+double positive cells $(14.53 \pm 16.88)$ was higher in SSc patients than in controls $(0.11 \pm 0.08,0.01 \pm 0.01,0.01 \pm 0.01$ and $0.39 \pm 0.40$, respectively) $(p<0.0001)$. A high percentage of HLA-G +cells $(30.47 \pm 26.75)$ was detectable on CD $4{ }^{\text {dull }} C D 8^{\text {high }}$ cells from SSc patients only.

Conclusions: These data indicate that in SSc secretion and/or shedding of SHLA-G and $\mathrm{mHLA}-\mathrm{G}$ are clearly elevated and involved in immune dysregulation.

\section{REFERENCES:}

[1] Gabrielli A, Avvedimento EV, Krieg T. Scleroderma. N Engl J Med 2009;360:1989-2003.

[2] Negrini S, Fenoglio D, Parodi A, Kalli F, Battaglia F, Nasi G, et al. Phenotypic Alterations Involved in CD8+ Treg Impairment in Systemic Sclerosis. Front Immunol 2017:8:18.

[3] Parel Y, Aurrand-Lions M, Scheja A, Dayer JM, Roosnek E, Chizzolini C. Presence of CD4+CD8+ double-positive T cells with very high interleukin-4 production potential in lesional skin of patients with systemic sclerosis. Arthritis Rheum 2007;56:3459-67.

Acknowledgements: This work was supported by "Gruppo Italiano Lotta alla Sclerodermia" (GILS). The sponsor had no role in study design, in the collection, analysis and interpretation of data, in the writing of the report, and in the decision to submit the article for publication.

Disclosure of Interest: None declared

DOI: 10.1136/annrheumdis-2018-eular.3241

\section{THU0408}

CHARACTERISTICS OF MILD TO MODERATE LUNG DISEASE IN SYSTEMIC SCLEROSIS AND IMPACT OF SURVIVAL; DATA FROM THE POPULATION-BASED, NATIONWIDE NORWEGIAN COHORT

H. Fretheim ${ }^{1}$, M. Seip ${ }^{2}$, A.-K. Halse ${ }^{3}$, H. Bitter ${ }^{4}$, M. Wallenius ${ }^{5}$, M. Aaløkken¹, T. Garen ${ }^{1}, \varnothing$. Midtvedt ${ }^{1}, \varnothing$. Molberg ${ }^{1}$, A.-M. Hoffmann-Vold ${ }^{1} .{ }^{1}$ Oslo University Hospital, Oslo; ${ }^{2}$ University Hospital of North Nonway, Tromso; ${ }^{3}$ Haukeland University Hospital, Bergen; ${ }^{4}$ Hospital of Southern Norway, Kristiansand; ${ }^{5}$ St Olav's University Hospital, Trondheim, Norway

Background: In systemic sclerosis (SSc) it is well-documented that severe interstitial lung disease (ILD), defined as an extent of lung fibrosis $>20 \%$ on high resolution computed tomography (HRCT) is strongly associated with male gender, anti-topoisomerase antibody (ATA), diffuse cutaneous (dc) form of disease and decreased survival. Much less is, however, known about the disease characteristics and impact of less lung involvement. A possible explanation might be the selected nature of many hitherto investigated SSc cohorts. In an era with novel treatment options, and multiple ongoing clinical trials, it is important to gain natural history knowledge on the whole spectre of lung involvement in systemic sclerosis. Objectives: Characterise the SSc patients with mild to moderate ILD, defined as $1 \%-10 \%$ extent of lung fibrosis on HRCT in the population based nationwide Norwegian SSc cohort and assess the impact of this ILD form on survival.

Methods: The Norwegian, nationwide SSc (Nor-SSc) study cohort included all adult SSc patients who were resident in Norway between 01.01.2000-01.01.2013 and met the classification criteria for SSc. Detailed electronic patient journal review was performed in all patients to assess demographic, clinical and ILD features at baseline. Pulmonary function tests (PFTs) and lung HRCT images were analysed and the extent of lung fibrosis was expressed as percentage of total lung volumes. We defined mild-moderate ILD as $1 \%-10 \%$ fibrosis on HRCT and severe disease as $>20 \%$ fibrosis. Ground glass opacities, bronchiectasis and honeycombing were registered if present. Vital status and causes of deaths were available in all patients at study end (January 2018). Descriptive statistics and regression analysis were applied.

Results: The Nor-SSc cohort included 815 patients. Baseline lung HRCT images were available in 416 patients $(51 \%)$, whereof $226(54.3 \%)$ had signs compatible with ILD. Mild to moderate ILD, defined as 1\%-10\% lung fibrosis were present in $149(65.9 \%)$, while $48(21.2 \%)$ had extensive lung fibrosis defined as $>20 \%$. Patients with mild-moderate ILD were 55.2 years at SSc onset and characterised by female gender (81.9\%) and limited cutaneous SSc $(70.5 \%)$. Their antibody profile was dominated by anti-centromere $\mathrm{Ab}$ (ACA) in $48.3 \%$, followed by anti-polymerase III, ATA, and anti-RNP, in $14.1 \%, 13.4 \%$ and $5.4 \%$, respectively. The mean modified Rodnan skin score (mRSS) was 9.3 (9.3\%), baseline FVC was $93.4 \%$ and DLCO $66.5 \%$. Univariable associations of clinical characteristics and mild-moderate ILD are shown in figure 1. After a mean observation time of 8.2 years, $49(32.9 \%)$ of patients with mild-moderate ILD had died. The 5- and 10 year survival was $76 \%$ and $62 \%$ compared to $83 \%$ and $76 \%$ in patients with no ILD $(p=0.03)$. In univariable cox regression analyses, mild-moderate ILD was associated with mortality (HR 1.6, 95\% Cl 1.03-2.40, $\mathrm{p}=0.034)$.

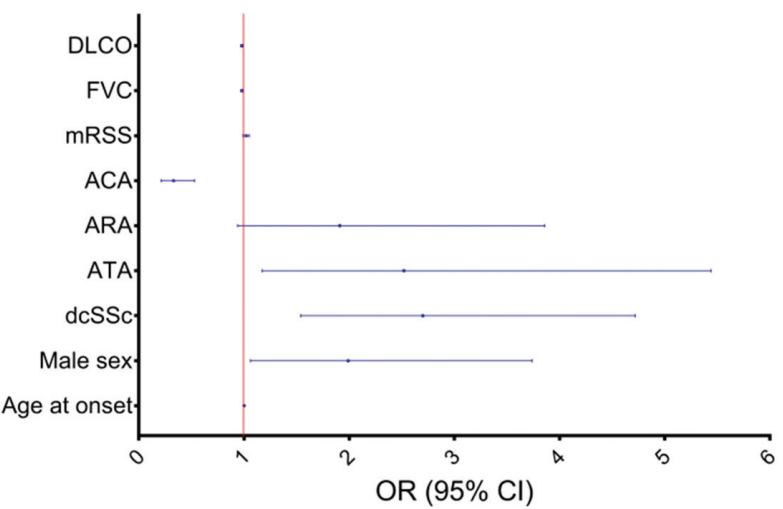

Abstract THU0408 - Figure 1. Associations of clinical characteristics and mild-moderate ILD at baseline

Conclusions: In this population based, nationwide study, mild-moderate ILD was frequent and had a considerably impact on survival in SSc patients.

Disclosure of Interest: None declared

DOI: 10.1136/annrheumdis-2018-eular.7121 